\title{
Usuários do CAPS II: nutrição e qualidade de vida
}

\author{
CAPS II users: nutrition and quality of life
}

\section{Vivian Carla Honorato dos Santos de Carvalho', Patricia Baier Krepsky², Ana Myllena Pinto Rocha ${ }^{3}$, Mariana Flores Silva dos Santos ${ }^{4}$}

\begin{abstract}
'Autora para correspondência. Universidade Federal da Bahia. Salvador, Bahia, Brasil. ORCID: 0000-0003-4000-0913. vihonorato@hotmail.com ${ }^{2}$ Universidade Federal da Bahia. Salvador, Bahia, Brasil. ORCID: 0000-0002-0112-2769. pkrepsky@gmail.com ${ }^{3}$ Universidade Federal da Bahia. Salvador, Bahia, Brasil. ORCID: 0000-0002-0817-2507. mydiscipula@hotmail.com ${ }^{4}$ Universidade Federal da Bahia. Salvador, Bahia, Brasil. ORCID: 0000-0001-6340-7121.mflores_48@hotmail.com
\end{abstract}

RESUMO I OBJETIVO: Avaliar o estado nutricional, o perfil clínico e a qualidade de vida (QV) de usuários do Centro de Atenção Psicossocial II (CAPS II) em Vitória da Conquista, BA. MÉTODOS: Foi realizado um estudo transversal com 120 usuários do CAPS II por meio de formulário contendo perguntas fechadas sobre dados sociodemográficos, qualidade de vida (QV) e autopercepção da saúde, assim como perguntas abertas sobre morbidade referida. Foi realizada também avaliação antropométrica (peso, altura e circunferência da cintura) para avaliar o estado nutricional. Para avaliação da $Q V$ foi usado o instrumento SF-12. A morbidade referida foi categorizada segundo a CID10. RESULTADOS: Os usuários apresentavam idade média de $39,8 \pm 12,48$ anos, $64,5 \%$ eram mulheres, $55,8 \%$ eram solteiros e $71,7 \%$ não estavam aposentados. Em relação à $Q V$, o componente mental sumarizado teve um resultado inferior quando comparado ao componente físico sumarizado. A autopercepção de saúde ruim alcançou $70,0 \%$ dos entrevistados; os transtornos mais prevalentes foram o transtorno de humor e esquizofrenia; $57,1 \%$ dos usuários apresentaram sobrepeso e obesidade e $65,5 \%$ dos participantes tiveram alteração da relação circunferência-estatura. CONCLUSÃO: Os usuários do CAPS II revelaram uma prevalência importante de excesso de peso, obesidade central, baixa qualidade de vida e uma autopercepção ruim da sua saúde. Compreender essas questões tornasse extremamente relevante para pensarmos em políticas locais de atendimento e organização dos CAPS, com uma maior interlocução com os serviços de atenção primária dos municípios, promovendo uma atenção integral e promotora de saúde.

PALAVRAS-CHAVE: Estado nutricional. Transtorno mental. Serviços de saúde mental. Obesidade.
ABSTRACT I AIM: Evaluating nutritional status, clinical profile and quality of life (QOL) of users on Psychosocial Care Center II (CAPS II), in Vitória da Conquista, Bahia. METHODS: A crosssectional study was carried out with 120 users of CAPS II using a form containing closed questions about sociodemographic data, quality of life (QOL) and self-perception of health, as well as open-ened questions about referral morbidity. An anthropometric evaluation (weight, height and waist circumference) was performed to evaluate the nutritional status. The instrument SF-1 2 was used to assess QOL. The referred morbidity was categorized according to ICD-10. RESULTS: The average age was $39.8 \pm$ 12.48 years, $64.5 \%$ of the participants were women, $55.8 \%$ were single and $71.7 \%$ were not retired. Regarding $Q O L$, the summarized mental component presented a lower result when compared to the summarized physical component. Poor selfperception of health was reported by $70 \%$ of interviewees. The most prevalent disorders of mood disorder and schizophrenia and $57.1 \%$ of the users were overweight and obese and $65.5 \%$ of the participants had a change in the circumference-height relationship. CONCLUSION: Users of CAPS II revealed an important prevalence of overweight, central obesity, poor quality of life and poor self-perception of health. Understanding these issues made it extremely relevant to think about local policies of care and organization of the CAPS, with a greater interlocution with the primary care services of the municipalities, promoting integral care and health promotion.

KEYWORDS: Nutritional status. Mental disorders. Mental health services. Obesity. 


\section{Introdução}

O Brasil é um dos países que apresentam maior prevalência de transtorno mental (TM) na população adulta, de 15-59 anos, revelando índices elevados para transtornos relacionados ao uso de substâncias psicoativas, transtornos do humor e transtornos da ansiedade (Santos \& Marluce, 2010), ressaltando que os TM são um importante problema de saúde pública. A estimativa da Organização Mundial da Saúde (OMS) evidencia que cerca de 450 milhões de pessoas sofram de algum tipo de transtorno mental, outros dados demonstram que os problemas de saúde mental são responsáveis por $8,8 \%$ e $16,6 \%$ do total da carga de doença relacionadas às condições de saúde em países de baixa e média renda, respectivamente (World Health Organization, 2002; Organização Mundial de Saúde, 2012).

Segundo a Classificação Internacional de Transtornos Mentais e de Comportamento da OMS (1996), TM podem ser alterações de humor associadas a uma angústia expressiva, produzindo alterações no desempenho físico e mental nos âmbitos pessoal, social, ocupacional e familiar. Esses transtornos são culturalmente estigmatizados, o que prejudica ainda mais o relacionamento social dos pacientes, comprometendo sua qualidade de vida (QV) (Freitas, Pinto, Nunes, Silva e Souza \& Machado, 2016).

Durante a década de 70, ocorreram, no Brasil, lutas representativas contra $\circ$ modelo hospitalocêntrico de atenção a indivíduos com sofrimento psíquico. Advindo da Reforma Psiquiátrica Brasileira, esses movimentos propuseram uma radical ruptura do modelo manicomial, a partir da construção de um novo paradigma científico, político e ético de cuidado. Nesse sentido, se antes apenas o manicômio existia como espaço de tratamento, após a reforma, houve a criação da Rede de Atenção Psicossocial, composta por serviços e equipamentos, tais como - Centro de Atenção Psicossocial (CAPS), Serviço Residencial Terapêutico, Núcleo de Assistência à Saúde da Família, Ambulatórios de Saúde Mental, além dos Centros de Convivência e outros espaços de socialização (Portaria $n^{\circ} 3.088$, de 23 de dezembro de 2011, 2011; Ribeiro Ramos \& Guimarães, 2011).
Os CAPS surgem como instituições brasileiras vinculadas ao Sistema Único de Saúde (SUS) com - objetivo de oferecer acolhimento humanizado, atendimento médico e psicológico que estimulem a integração social por meio de atividades diversificadas. A exemplo disto tem-se o atendimento individual e em grupos, oficinas terapêuticas executadas por profissional de nível superior ou nível médio, visitas domiciliares e atividades comunitárias focando a integração do paciente na comunidade e sua inserção familiar e social (Zórtea, Bock, Moreno, \& Abreu, 2009; Portaria $\mathrm{n}^{\circ} 336$, de 19 de fevereiro de 2002, 2002).

Os indivíduos que participam desse serviço podem apresentar QV ruim, decorrente da gravidade do transtorno, dos efeitos colaterais dos medicamentos e dos eventos estressores que interferem na evolução do problema. Além disso, a presença de síndrome metabólica que é considerada um dos principais fatores de risco para doenças cardiovasculares em pessoas com esquizofrenia,e também pode estar associada a uma pior QV (Freitas et al., 2016).

Apesar de ser um grupo de alto risco de síndrome metabólica, há poucos estudos que descrevem os aspectos nutricionais do grupo de usuários do CAPS. Vale ressaltar que dentro dos objetivos gerais da Rede de Atenção Psicossocial não são observadas ações referentes à prevenção dos agravos metabólicos associados à obesidade, bem como o seu diagnóstico. Destacando-se que o levantamento de dados que possa auxiliar no planejamento das ações e na adequação do cuidado de saúde mental, bem como em identificar fatores que influenciam a QV é de fundamental importância para auxiliar no planejamento de intervenções nos serviços de saúde (Freitas et al., 2016). Dessa forma, este estudo tem como objetivo avaliar o estado nutricional, perfil clínico e qualidade de vida dos usuários que são atendidos no Centro de Atenção Psicossocial II, no município de Vitória da Conquista, Bahia. 
Métodos

O presente estudo foi descritivo do tipo transversal, realizado entre novembro de 2016 a junho de 2017, em Vitória da Conquista, Bahia. Foram entrevistados 120 usuários do CAPS II com idade superior a 18 anos e a seleção da amostra se deu por conveniência. Participaram do estudo todos os usuários que estavam em atendimento no CAPS II no referido período, sendo entrevistados os que aceitaram livremente a sua participação após explicação verbal sobre a pesquisa. Pessoas que não fossem usuários do CAPS II e que não aceitassem participar livremente do estudo foram consideradas inelegíveis para pesquisa, assim como usuários que não apresentavam condições de responder ao questionário.

Antes da coleta de dados, toda a equipe foi treinada, com explicações sobre a metodologia do estudo, aplicação dos questionários, condutas no trabalho de campo e realização das medidas antropométricas. A coleta de dados ocorreu na sala de atendimento do CAPS II, nos turnos matutino e vespertino.

Inicialmente foi aplicado formulário composto por perguntas fechadas e abertas, conforme especificado abaixo, estruturado em quatro blocos aplicados num mesmo momento na seguinte sequência: dados sociodemográficos, autopercepção da saúde, QV e problemas de saúde. Em seguida foi realizada avaliação antropométrica.

\section{Avaliação das características sociodemográfica:}

Dentro das variáveis sociodemográficas (escolaridade, sexo, idade, estado conjugal, número de filhos e aposentadoria), a escolaridade foi categorizada em: (a) analfabeto, (b) ensino fundamental incompleto, (c) ensino fundamental completo, (d) ensino médio, (e) superior incompleto e (f) superior completo; o estado conjugal foi categorizado em: (a) casado/ companheiro, (b) solteiro, (c) separado/divorciado e (d) viúvo; e o número de filhos foi categorizado em: (a) 0, (b) 1 a 4 filhos e (c) 5 filhos ou mais.

\section{Avaliação da autopercepção de saúde:}

A autopercepção de saúde foi obtida por meio da pergunta: "Em geral, você diria que sua saúde está:". As seguintes opções de respostas foram fornecidas ao usuário: (a) excelente, (b) muito boa, (c) boa, (d) regular e (e) ruim. Para avaliar a autopercepção da saúde dos usuários nesse estudo, a variável foi dicotomizada em autopercepção boa ou ruim de saúde. A combinação das categorias excelente, muito boa e boa indicou uma boa autopercepção de saúde, e a combinação das categorias regular e ruim indicou uma autopercepção ruim de saúde (Darviri, Artemiadis, Tigani, Alexopoulos, 2011).

\section{Avaliação da qualidade de vida:}

Para avaliação da $Q V$, foi usado o instrumento genérico de aferição de qualidade de vida, 12Item Short-Form Health Survey (SF-12). Constituído por doze itens derivados do 36-Item Health Survey (SF-36), o SF-1 2 avalia oito diferentes dimensões de influência sobre a $Q V$, considerando a percepção do indivíduo em relação aos aspectos de sua saúde nas últimas quatro semanas. Cada item do questionário possui um grupo de respostas distribuídas em uma escala graduada, sendo avaliadas as seguintes dimensões: função física, aspecto físico, dor, saúde geral, vitalidade, função social, aspecto emocional e saúde mental. Através de um algoritmo próprio do instrumento, dois escores podem ser mensurados: o físico (Physical Component Summary ou PCS) e - mental (Mental Component Summary ou MCS). Em ambos, a pontuação varia em uma escala de zero a cem, sendo os maiores escores associados a melhores níveis de QV (Silveira, Almeida, Freire, Haikal, \& Martins, 2013).

\section{Avaliação dos problemas de saúde:}

A avaliação dos problemas de saúde foi realizada através das seguintes questões: (1) Qual é o principal problema de saúde ou doença que limita suas atividades? (2) Qual é o transtorno neuropsiquiátrico diagnosticado pelo CAPS? 
Considerando as respostas obtidas a CID-10, os problemas de saúde relatados pelos pacientes como limitadores de suas atividades (morbidade referida) e os transtornos diagnosticados foram categorizados em: (a) esquizofrenia e outros transtornos psicóticos; (b) transtorno de humor; (c) transtorno de ansiedade; (d) transtornos de personalidade; (e) delirium e demência; ( $f$ ) transtorno do sono; $(g)$ depressão; $(h)$ dores e (i) outros diagnósticos.

\section{Avaliação antropométrica:}

Foram realizadas as seguintes medidas antropométricas: peso, altura e circunferência da cintura, sendo todas realizadas em duplicata. $O$ peso foi verificado em balanças portáteis (Marte, LC200pp, Marte, São Paulo, Brasil) com indivíduos descalços, ereto, com pés juntos, braços juntos ao corpo e com o mínimo de roupas. A altura, medida através de estadiômetro vertical, sobre o qual os pacientes foram posicionados descalços no centro do equipamento, com cabeças livres de adereços, de pé, eretos, com braços estendidos ao longo do corpo. A circunferência da cintura $(\mathrm{CC})$ foi medida por fita métrica inelástica, posicionada na cintura natural, ponto médio entre o último arco costal e a crista ilíaca (World Health Organization, 2011).

O estado nutricional foi realizado por meio do Índice de Massa Corporal (IMC), definido como a relação entre o peso em quilos e a altura em metros elevado ao quadrado $\left(\mathrm{kg} / \mathrm{m}^{2}\right)$, sendo categorizado em: (a) $<18,5$ como baixo peso; (b) 18,5 a 25,0 como eutrofia; (c) 25,0 a 29,9 como sobrepeso ou pré-obeso e (d) $>30,0$ como obesidade 12. A circunferência da cintura foi categorizada em: (a) $>94 \mathrm{~cm}$ para homens e $>80 \mathrm{~cm}$ para mulheres para risco aumentado de complicações metabólicas; (b) $>102 \mathrm{~cm}$ para homens e $>88 \mathrm{~cm}$ para mulheres para risco muito aumentado de complicações metabólicas (WHO, 2011). A relação cintura-estatura (RCE) foi obtida pelo quociente entre a circunferência da cintura $(\mathrm{cm})$ e a estatura $(\mathrm{cm})$, tendo como ponto de corte o valor de 0,5 , ou seja, a cintura deverá ser menor que a metade da altura. Foi categorizado com risco metabólico os indivíduos com $\mathrm{RCE} \geq 0,5$ (Lópes-Sobaler et al., 2016).
Além disso, definimos um índice composto combinando IMC e RCE, conforme estabelecido por López-Sobaler et al. (2016). O índice é composto por uma variável de cinco categorias, onde o Nível 1 representa a melhor situação antropométrica e o Nível 5 representa a pior situação antropométrica. Os cinco grupos deste índice composto são: (a) Nível 1: peso abaixo do peso ou normal e $\operatorname{RCE}<0,5$; (b) Nível 2: sobrepeso ou obesidade e RCE <0,5; (c) Nível 3: peso abaixo do peso ou normal e RCE $\geq 0,5$; (d) Nível 4: excesso de peso e RCE $\geq 0,5$ e (e) Nível 5: obesidade e RCE $\geq 0,5$.

Para análise estatística dos dados foi utilizado - programa SPSS (Statistical Package for Social Science), versão 20.0 para Windows por meio de frequências absolutas e relativas (\%). Foi realizado o teste de Shapiro-Wilk para avaliar se a amostra possuía distribuição normal. Foram utilizados os testes do Qui-quadrado de Pearson, para comparação entre proporções e o Teste t-Student para avaliar diferenças entre as médias. As características da amostra foram descritas em termos de porcentagem, média e desvio padrão (DP) ou intervalo de confiança de $95 \%$.

O presente estudo foi desenvolvido respeitando os princípios básicos da bioética que implica a pesquisa envolvendo seres humanos como: respeito a autonomia, beneficência, não-maleficência e justiça que estão explicitados na Resolução CNS $n^{\circ}$ 466/12. O estudo foi aprovado pelo Comitê de Ética e Pesquisa do IMS/CAT - UFBA (Parecer: 1.899.069) e todos os participantes envolvidos assinaram 0 Termo de Consentimento Livre e Esclarecido.

\section{Resultados}

\section{Caracterização sociodemográfica}

A amostra do estudo foi composta por 120 indivíduos, com idade média de 39,8 anos ( $D P=12,5$ ), sendo $64,5 \%$ do sexo feminino. A tabela 1 descreve as características dos usuários do CAPS, destacando que $39,2 \%$ possuíam ensino fundamental incompleto e $38,4 \%$ ensino superior incompleto a completo, $55,8 \%$ eram solteiros, $31,6 \%$ não tinham filhos e $71,7 \%$ não estavam aposentados. 
Tabela 1. Características dos usuários do Centro de Atenção Psicossocial Il de Vitória da Conquista, Bahia, [N (\%) ou média (DP)], 2017 (continua)

\begin{tabular}{|c|c|c|c|c|}
\hline & $\begin{array}{c}\text { Total } \\
N=120\end{array}$ & $\begin{array}{l}\text { Feminino } \\
\mathrm{N}=77\end{array}$ & $\begin{array}{l}\text { Masculino } \\
\qquad \mathrm{N}=43\end{array}$ & $\begin{array}{c}\text { Valor } \\
\mathrm{P}\end{array}$ \\
\hline Sexo & $120(100 \%)$ & $77(64,5 \%)$ & $43(35,5 \%)$ & \\
\hline Idade (anos) & $39,8(\mathrm{DP}=12,5)$ & $42,52(\mathrm{DP}=12,1)$ & $35,02(\mathrm{DP}=11,8)$ & 0,001 \\
\hline Escolaridade & & & & 0,230 \\
\hline Analfabeto & $05(04,2 \%)$ & $05(06,5 \%)$ & $00(00,0 \%)$ & \\
\hline Ensino Fundamental Incompleto & $47(39,2 \%)$ & $25(32,5 \%)$ & $22(51,2 \%)$ & \\
\hline Ensino Fundamental Completo & $17(14,2 \%)$ & $11(14,3 \%)$ & $06(14,0 \%)$ & \\
\hline Ensino Médio Completo & $05(04,2 \%)$ & $03(03,9 \%)$ & $02(04,7 \%)$ & \\
\hline Ensino Superior Incompleto & $44(36,7 \%)$ & $32(41,6 \%)$ & $12(27,9 \%)$ & \\
\hline Ensino Superior Completo & $02(01,7 \%)$ & $01(01,3 \%)$ & $01(02,3 \%)$ & \\
\hline Situação Conjugal & & & & 0,000 \\
\hline Solteiro/não casou & $67(55,8 \%)$ & $32(41,6 \%)$ & $35(81,4 \%)$ & \\
\hline Casado/companheiro & $41(34,2 \%)$ & $36(46,8 \%)$ & $05(11,6 \%)$ & \\
\hline Divorciado & $10(08,3 \%)$ & $07(09,1 \%)$ & $03(07,0 \%)$ & \\
\hline Viúvo & $02(01,7 \%)$ & $02(02,6 \%)$ & $00(00,0 \%)$ & \\
\hline Aposentado & & & & 0,730 \\
\hline $\operatorname{Sim}$ & $34(28,3 \%)$ & $21(27,3 \%)$ & $13(30,2 \%)$ & \\
\hline Não & $86(71,7 \%)$ & $56(72,7 \%)$ & $30(69,8 \%)$ & \\
\hline Filhos & & & & 0,107 \\
\hline 0 & $18(31,6 \%)$ & $10(23,8 \%)$ & $08(53,3 \%)$ & \\
\hline $1-4$ & $34(59,6 \%)$ & $28(66,7 \%)$ & $06(40,0 \%)$ & \\
\hline 5 ou mais & $05(08,8 \%)$ & $04(09,5 \%)$ & $01(06,7 \%)$ & \\
\hline \multicolumn{5}{|l|}{ SF-12 } \\
\hline $\mathrm{PCS}^{1}$ & $42,0(\mathrm{DP}=10,1)$ & $41,1(\mathrm{DP}=09,9)$ & $43,6(D P=10,4)$ & 0,197 \\
\hline $\mathrm{MCS}^{2}$ & $36,9(\mathrm{DP}=13,1)$ & $34,0(\mathrm{DP}=13,1)$ & $42,0(\mathrm{DP}=11,6)$ & 0,001 \\
\hline Morbidade Referida & & & & 0,202 \\
\hline $\mathrm{F} 20-29^{3}$ & $17(14,5 \%)$ & $07(09,1 \%)$ & $10(25,0 \%)$ & \\
\hline F30-394 & $31(26,5 \%)$ & $24(31,2 \%)$ & $07(17,5 \%)$ & \\
\hline $\mathrm{F} 40-49^{5}$ & $16(13,7 \%)$ & $10(13,0 \%)$ & $06(15,0 \%)$ & \\
\hline $\mathrm{R} 52^{\circ}$ & $09(07,7 \%)$ & $08(10,4 \%)$ & $01(02,5 \%)$ & \\
\hline Outros & $29(24,8 \%)$ & $19(24,7 \%)$ & $10(25,0 \%)$ & \\
\hline Sem Problema & $15(12,8 \%)$ & $09(11,7 \%)$ & $06(15,0 \%)$ & \\
\hline
\end{tabular}




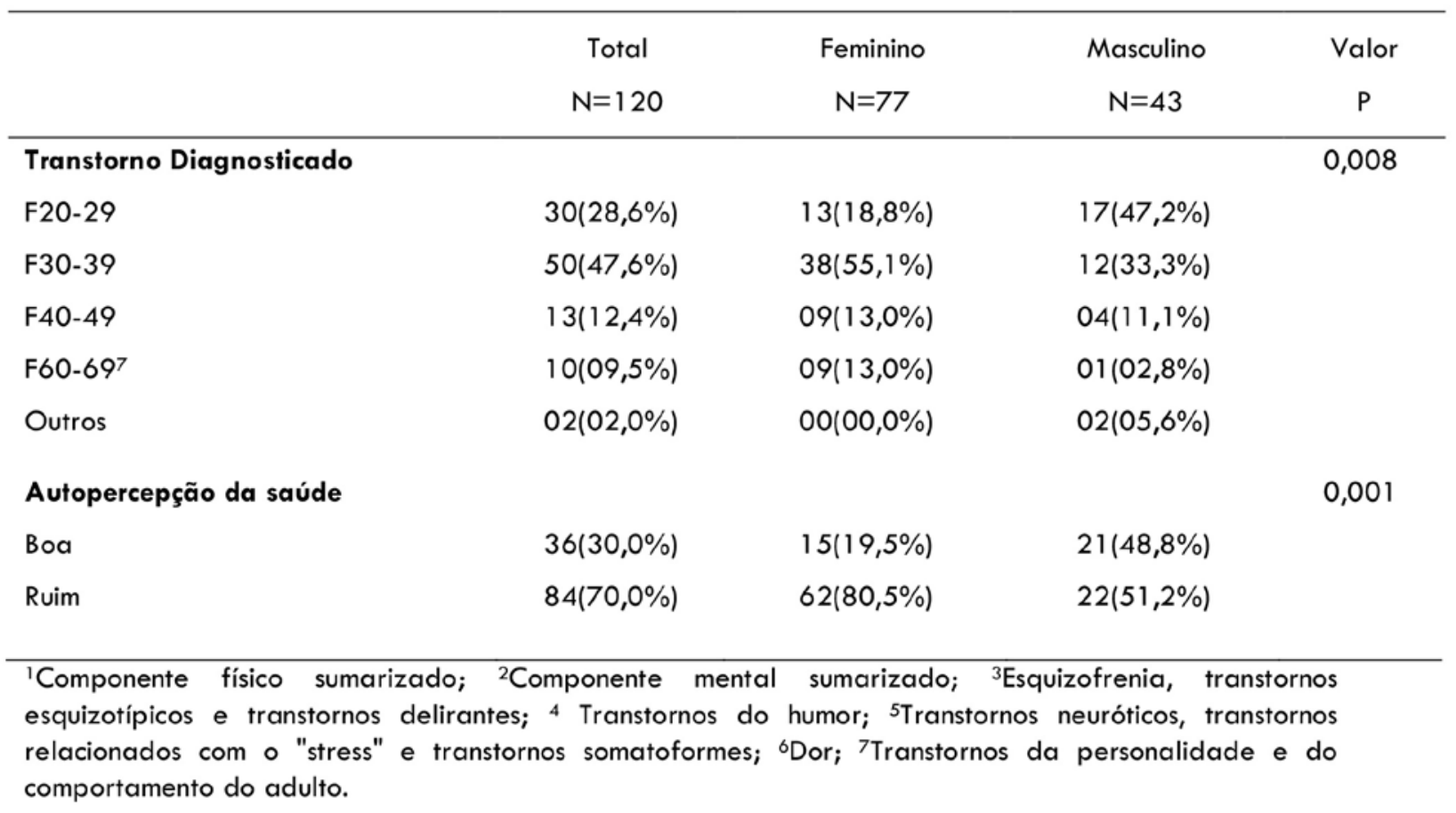

\section{Avaliação da autopercepção de saúde}

Ao avaliar a autopercepção de saúde, 70,0\% classificou sua saúde como ruim, com resultado significativamente pior para mulheres $(80,5 \%$; $p=0,001$ ) (Tabela 1).

\section{Avaliação da qualidade de vida}

Em relação à qualidade de vida, o MCS teve um resultado inferior $[36,9 \mathrm{DP}=13,1)]$, quando comparado com o PCS $[42,0(D P=10,1)]$. Destacase que as mulheres apresentaram um resultado significativamente menor em relação ao componente mental sumarizado $[34,0(D P=13,1) ; p=0,001]$, em comparação aos homens (Tabela 1).

\section{Avaliação dos problemas de saúde}

A frequência de problemas de saúde referidos foi similar entre os sexos, com predomínio de transtorno de humor $(26,5 \%)$, ressaltando que $12,8 \%$ dos usuários relataram ausência de problemas de saúde. Em relação ao transtorno diagnosticado pelo CAPS II, a maior prevalência foi o transtorno de humor $(47,6 \%)$, seguido de esquizofrenia $(28,6 \%)$ (Tabela 1$)$.

\section{Avaliação antropométrica}

Observa-se uma prevalência de $57,1 \%$ dos usuários com sobrepeso e obesidade. Em relação à circunferência da cintura, a prevalência foi de $57,2 \%$ de usuários com risco aumentado para complicações metabólicas, sendo mais freqüente entre as mulheres $(68,5 \% ; p=0,002)$. Analisando a RCE percebe-se uma alteração em $65,5 \%$ dos participantes do estudo, sem diferença significativa entre os sexos (Tabela 2). 
Tabela 2. Estado Nutricional dos usuários do Centro de Atenção Psicossocial Il de Vitória da Conquista, Bahia, [N (\%) ou média (DP)], 2017

\begin{tabular}{|c|c|c|c|c|}
\hline & $\begin{array}{c}\text { Total } \\
\mathrm{N}=120\end{array}$ & $\begin{array}{c}\text { Feminino } \\
N=77\end{array}$ & $\begin{array}{c}\text { Masculino } \\
\mathrm{N}=43\end{array}$ & $\begin{array}{c}\text { Valor } \\
\text { P }\end{array}$ \\
\hline $\mathrm{CC}^{1}$ & $86,3 \pm 13,69$ & $85,91 \pm 13,60$ & $87,10 \pm 13,97$ & 0,652 \\
\hline $\mathrm{RCE}^{2}$ & $00,54 \pm 00,09$ & $00,56 \pm 00,09$ & $00,51 \pm 00,09$ & 0,007 \\
\hline $\begin{array}{l}I M C^{3} \\
I M C\end{array}$ & $26,89 \pm 05,60$ & $27,65 \pm 05,86$ & $25,36 \pm 04,80$ & $\begin{array}{l}0,025 \\
0,385\end{array}$ \\
\hline Desnutrido & $06(05,0 \%)$ & $03(03,9 \%)$ & $03(07,0 \%)$ & \\
\hline Eutrófico & $45(37,8 \%)$ & $27(35,5 \%)$ & $18(41,9 \%)$ & \\
\hline Sobrepeso & $35(29,4 \%)$ & $21(27,6 \%)$ & $14(32,6 \%)$ & \\
\hline Obesidade & $33(27,7 \%)$ & $25(32,9 \%)$ & $08(18,6 \%)$ & \\
\hline Índice Composto & & & & 0,221 \\
\hline Nível 1 & $36(30,3 \%)$ & $18(23,7 \%)$ & $18(41,9 \%)$ & \\
\hline Nível 2 & $03(02,5 \%)$ & $02(02,6 \%)$ & $01(02,3 \%)$ & \\
\hline Nível 3 & $14(11,8 \%)$ & $11(14,5 \%)$ & $03(07,0 \%)$ & \\
\hline Nível 4 & $32(26,9 \%)$ & $20(26,3 \%)$ & $12(27,9 \%)$ & \\
\hline Nivel 5 & $34(28,6 \%)$ & $25(32,9 \%)$ & $09(20,9 \%)$ & \\
\hline RCE & & & & 0,093 \\
\hline Normal & $41(34,5 \%)$ & $22(28,9 \%)$ & $19(44,2 \%)$ & \\
\hline Risco Metabólico & $78(65,5 \%)$ & $54(71,1 \%)$ & $24(55,8 \%)$ & \\
\hline $\mathrm{CC}$ & & & & 0,002 \\
\hline Normal & $51(42,9 \%)$ & $24(31,6 \%)$ & $27(62,8 \%)$ & \\
\hline Risco & $24(20,2 \%)$ & $16(21,1 \%)$ & $08(18,6 \%)$ & \\
\hline Risco Elevado & $44(37,0 \%)$ & $36(47,4 \%)$ & $08(18,6 \%)$ & \\
\hline
\end{tabular}

${ }^{1}$ Circunferência da cintura; ${ }^{2}$ Relação cintura e estatura; ${ }^{3}$ Índice de massa corporal.

Ao se avaliar o índice composto, percebe-se que apenas $30,3 \%$ dos participantes apresentaram uma situação antropométrica ótima, com maior prevalência no sexo masculino $(41,9 \%)$, enquanto que $55,5 \%$ dos usuários apresentaram excesso de peso e obesidade, não havendo diferenças significativas entre os sexos.

\section{Discussão}

A população estudada, em sua maioria, era feminina, solteira, com baixa escolaridade e não aposentada. De forma majoritária, os dados da literatura demonstraram que as mulheres apresentam maior prevalência de TM em relação aos homens (Santos \&Marluce, 2010; Coutinho, Matijavesich, Scazufca, 
\& Menezes, 2014). O estudo realizado nos CAPS da Região Sul do Brasil, revelou que dos 1493 usuários entrevistados, $59,2 \%$ era do sexo feminino (Franzmann et al., 2017). Apesar disso, mesmo havendo um significativo número de estudos que abordem essa temática, ainda não se consolidou - motivo pelo qual exista predomínio de mulheres nesses serviços. Entretanto, é válido ressaltar que são as mulheres quem mais procuram os serviços de saúde, quando comparadas aos homens homens (Rocha, Almeida, Araúio, \& Virtuoso, 2010). Além disso, nas últimas décadas, muitas mudanças ocorreram no papel da mulher, algo que propiciou sua maior inserção no mercado de trabalho com subseqüente acúmulo de tarefas, o que pode ter contribuído, dessa maneira, com o surgimento e a maximização dos problemas de saúde mental nessa população (Scholl et al., 2017).

Observou-se também que a maioria dos usuários CAPS II possuíam escolaridade de ensino fundamental incompleto ou completo ou nenhuma escolaridade. Esse fato foi correlacionado com maior risco de apresentar TMC e está de acordo com ○ que foi abordado por Coutinho (2014), quando descreve que quanto maior a escolaridade dos usuários menor a prevalência de TMC. De igual modo, outros dois estudos demonstraram correlação entre indivíduos com TMC com baixa escolaridade, com prevalência de $74 \%$ em estudo desenvolvido em São Paulo (Coutinho et al., 2014) e 53\% em estudo realizado na Bahia(Rocha et al., 2010). Por outro lado, nesse estudo percebeu-se prevalência de $38,4 \%$ de usuários com escolaridade referente ao ensino superior incompleto ou completo, o que não é comumente encontrado nessa população, que apresenta nível de instrução entre baixo e médio (Franzmann et al., 2017; Carvalho et al., 2017).

Houve também comprometimento da QV desses usuários os quais apresentaram $\circ$ MCS com um resultado inferior ao PCS. Vale destacar que as mulheres apresentaram um resultado significativamente menor na qualidade de vida em relação ao componente mental sumarizado $[34,0(D P=13,10) ; p=0,001]$ quando comparado com o sexo masculino $[42,0(D P=11,63)]$. Esse resultado foi semelhante a estudo realizado com indivíduos diagnosticados com transtorno obsessivo compulsivo (TOC), o qual demonstrou que homens apresentam melhor qualidade de vida nos domínios físico ( $p=0,005)$ e mentais ( $p$ $=0,001$ ) comparados às mulheres (Scholl et al., 2017), sendo necessária uma atenção direcionada as mulheres em sofrimento psíquico.

A baixa $Q V$ relacionada aos usuários do CAPS pode estar associada também ao sedentarismo, à dependência funcional, à me dicação, à baixa renda e escolaridade, uma vez que essas variáveis se apresentaram associadas a uma $Q V$ comprometida (Freitas et al., 2016). Em contrapartida, os indivíduos casados, com filhos, com renda familiar acima de três salários mínimos e que praticavam atividades físicas foram relacionados à uma melhor QV(Cardoso et al., 2006).

De modo geral os usuários do CAPS apresentam distúrbios mais graves comparado à pacientes capazes de manter seu tratamento ambulatorial (Cavalcanti, Dahl, Carvalho \&Valencia, 2009), esse fato pode explicar a prevalência da $Q V$ comprometida. É importante entender esses fatores, que levam ao agravo do problema, pois eles podem ser a chave para melhor adaptação do indivíduo minimizando seu sofrimento e o impacto da doença melhorando assim sua QV(Scholl et al., 2017)..

Ao avaliar a autopercepção de saúde $70,0 \%$ dos entrevistados a classificou como ruim, o que pode ser justificado pelos mesmos fatores que afetaram a QV dos usuários. Este importante resultado demonstra que esses indivíduos não estão satisfeitos com sua saúde, além disso, observa-se a relevância da avaliação feita pelos próprios usuários para que ocorra aprimoramento de tratamentos psicológicos, promovendo que o mesmo seja parte do processo de mudança e intervenha no espaço no qual está inserido, sendo observado que essa tendência tem sido destacada na literatura da área, nos últimos anos (Cesari \& Bandeira, 2010).

Os dados do presente estudo revelaram indivíduos com predomínio de transtornos de humor e esquizofrenia tanto para morbidade autorreferida quanto para transtornos diagnosticados pelo próprio CAPS II. Isto está de acordo com os dados publicados pelo Consórcio Internacional de Epidemiologia Psiquiátrica (ICPE) da OMS que revelou que há no Brasil índices elevados 
de transtornos de humor e ansiedade (WHO, 2000). Outro estudo realizado na cidade de São Paulo mostrou que o transtorno de ansiedade foi - distúrbio mais comum, afetando $19,9 \%$ dos participantes, seguido pelo distúrbio de humor com prevalência de 11\% (Viana \& Andrade, 2012). Pesquisa realizada no CAPS (tipos I, II e III) buscou avaliar o impacto da participação dos usuários em atividades físicas e quais foram às mudanças em aspectos de suas vidas a partir de sua entrada no CAPS. Evidenciou-se que o transtorno depressivo foi o mais prevalente, seguido de esquizofrenia e transtorno bipolar (Franzmann et al., 2017).

Diante do exposto, pesquisas sobre esquizofrenia tem sido cada vez mais importantes pelo fato de esse considerável transtorno ser uma das dez principais causas de incapacitação em todo o mundo. Vale ressaltar ainda que dentro dessas principais causas de incapacitação, cinco delas estão associadas com TM e com esquizofrenia, seguidamente associada em menor grau à depressão e aos transtornos bipolares (Santos \& Siqueira, 2010). No campo dos DALY - Disability Adjusted Life Years ou Anos de vida perdidos ajustados por incapacidade, destaca-se a carga das das doenças crônicas não transmissíveis em todas as regiões do Brasil, em particular as doenças cardiovasculares, os transtornos mentais, o diabetes e a doença pulmonar obstrutiva crônica, sendo a depressão a principal causa de carga de doenças entre as mulheres demonstrando a relevância da temática para a saúde pública (Leite et al, 2015). Entretanto, é importante ressaltar que $12,8 \%$ dos usuários relataram ausência de problemas de saúde, entretanto esses usuários possuem transtorno mental diagnosticado pelo CAPS, o que demonstra a necessidade de novos estudos com esse público, buscando compreender a complexidade referente à percepção do indivíduo sobre si e sobre a sua saúde.

Os transtornos mentais têm sido caracterizados em muitos estudos pela associação com IMC mais elevado e nos resultados da presente investigação a maioria dos usuários do CAPS encontra-se com sobrepeso e obesidade (Zórtea, Guimarães, Gama, \& Belmonte-de-Abreu, 2010). Resultados semelhantes são encontrados na literatura nas quais pacientes que possuem distúrbios mentais e fazem uso de medicamentos controlados apresentam- se com sobrepeso, $39,6 \%$ e $46,2 \%$ e obesidade, $33 \%$ e $25,6 \%$ (Leitão-Azevedo et al., 2006; Zórtea et al., 2009). Todo esse problema pode estar associado a fatores próprios do grupo como o uso de medicamentos que, em sua maioria, possuem efeitos colaterais de ganho de peso, aumento de apetite e dislipidemia e também outros fatores como o sedentarismo, genética, ambiente, estilo de vida e fatores emocionais (Kengeriski, Oliveira \& Delgado, 2014; Associação Brasileira para o Estudo da Obesidade e da Síndrome Metabólica, 2016).

A obesidade é uma doença complexa e multifatorial que está associada a diversos agravos e tem se tornado um problema de saúde pública mundial devido ao aumento de sua prevalência em vários países, sendo uma preocupação importante em pacientes psicóticos em razão do risco de diabetes melito e doenças cardiovasculares (Attux, Martini, Reis, \& Bressan, 2009; Medeiros, Possas \& Valadão Júnior, 2018). É possível observar que o transtorno mental tem grande impacto na saúde desses pacientes pelo fato de essa doença ser um estimulador para o desenvolvimento da obesidade (Verdolin, Sá, Guedes, Silva, \& Belmonte, 2012).

Esses dados são tão evidentes que houve redução dos índices de depressão com a perda de peso, apoiando a hipótese que a obesidade severa agrava a depressão. Esse estudo não excluiv, porém, a hipótese de que a obesidade pode ser causada ou agravada pela depressão (Verdolin et al, 2012). A análise de 560 pacientes americanos portadores de transtornos psiquiátricos graves, constatou a prevalência de obesidade muito superior à da população local (Susce, Villanueva, Diaz \& de Leon, 2005), ou seja, tratar a obesidade pode ser uma estratégia relevante para maior controle da doença.

Em relação à circunferência da cintura (CC) ० presente estudo teve prevalência de $57,2 \%$ com risco e alto risco de desenvolver doença cardiovascular, apontando uma maior prevalência entre as mulheres. Estudos realizados em Porto Alegre em diferentes períodos verificam alta prevalência de circunferência abdominal acima do desejado e um dos estudos destacam que a maior prevalência permanece entre as mulheres (Kengeriski et al, 2014; Zórtea et al., 2009). É relevante a recomendação da aferição da gordura 
abdominal nos usuários do CAPS, visto que esse tem sido indicador de obesidade abdominal (Cristóvão, Sato, \& Fujimori, 2011) e é um dos vários métodos descritos na literatura que tem uso preconizado pela praticidade, baixo custo e precisão diagnóstica (Corrêa, Tomasi, Thumé, Oliveira, \&Facchini, 2017).

Outra forma de verificar a presença de obesidade abdominal é através da RCE os valores acima do ponto de corte se relaciona com o risco de doenças cardiovascular, sua utilização tem sido relevante nas avaliações antropométricas. Em estudo feito na Espanha com indivíduos saudáveis, usando esse mesmo índice, foi observado uma prevalência de $58,4 \%$, sendo que apenas $36,1 \%$ da população apresentava uma ótima situação antropométrical 14. Entretanto, ainda que o IMC seja um método simples e prático para identificar casos de sobrepeso e obesidade o RCE tem-se mostrado melhor preditor de risco de doença ou mortalidade em relação ao IMC, pois consegue corrigir a altura e evitar a subestimação do risco em indivíduos que são mais alto ou mais baixo do que a média (López-Sobaler et al., 2016; Zhang et al., 2013).

Observou-se ainda outra medida antropométrica, o índice composto, o qual representa o estado nutricional do indivíduo, pois leva-se em consideração a classificação do IMC e a adiposidade central. Percebeu-se, por meio dele, que apenas $30,3 \%$ dos usuários do CAPS avaliados apresentaram uma situação antropométrica ótima e os homens se destacaram com maior prevalência, $41,9 \%$. Esse mesmo resultado foi encontrado em estudo desenvolvido na Espanha com indivíduos saudáveis (López-Sobaler et al., 2016). Não foram encontrados estudos usando esses parâmetros em usuários do CAPS, sendo um instrumento interessante para diagnóstico nutricional que poderia ser inserido na rotina do serviço.

No presente estudo, todos os índices se apresentaram elevados, o que comprova que esse grupo de pacientes se encontra, geralmente, acima do peso recomendado. É importante lembrar que as medidas antropométricas utilizadas neste estudo - IMC, CC e RCE - conseguiram, de forma significativa, correlacionar suas medidas com alto risco de doenças cardiovasculares. Esses resultados destacaram a tendência que esses pacientes possuem ao sobrepeso, à obesidade e também à gordura abdominal, demonstrando a necessidade de uma interferência imediata para retardar ou reverter essa tendência. Para isso, é necessário o devido acompanhamento nutricional e dietoterápico dos pacientes, para que ocorra avaliação dos hábitos alimentares e melhora do perfil nutricional (López-Sobaler et al., 2016). Os limites desse estudo estão relacionados ao desenho transversal, que não permite correlacionar causa e efeito, além disso, pela característica de funcionamento do CAPS II, foi necessária uma amostra por conveniência.

O presente estudo demonstrou que a baixa $Q V$ apresentada pelos pacientes deve fomentar ações que promovam a reabilitação psicossocial. Fazse necessário também a estruturação de grupos terapêuticos permanentes que abordem aspectos referentes à nutrição dentro do CAPS, com orientações para prevenção dos agravos e redução dos altos índices de adiposidade, com enfoque na educação alimentar e nutricional. Em suma, é importante promover estudos qualitativos que ampliem discussões sobre essas questões e estruturar o real fortalecimento do elo entre a atenção básica e o CAPS no atendimento integral e participativo dos usuários desses serviços.

\section{Agradecimentos}

À Coordenação de Saúde Mental do município de Vitória da Conquista, Bahia e a toda equipe do CAPS II pelo apoio e cordialidade sempre presente durante todo o período da pesquisa.

À Coordenação de Ações Afirmativas, Educação e Diversidade da Pró-Reitora de Ações Afirmativas e Assistência Estudantil pela bolsa de iniciação científica a aluna participante do estudo.

\section{Contribuições dos autores}

Carvalho, V. C. H. S participou da idéia e concepção da pesquisa, orientação, análise dos dados, redação, revisão, formatação do texto e encaminhamento do artigo. Krepsky, P. B. participou da redação e revisão do artigo. Rocha, A. M. P. participou da análise dos dados da pesquisa, redação, revisão e formatação do texto. Santos, M. F. S. participou da idéia e concepção da pesquisa, coleta dos dados e revisão. 


\section{Conflitos de interesses}

Nenhum conflito financeiro, legal ou político envolvendo terceiros (governo, empresas e fundações privadas, etc.) foi declarado para nenhum aspecto do trabalho submetido (incluindo mas não limitandose a subvenções e financiamentos, conselho consultivo, desenho de estudo, preparação de manuscrito, análise estatística, etc).

\section{Referências}

Associação Brasileira para o Estudo da Obesidade e da Síndrome Metabólica (2016). Diretrizes brasileiras de obesidade (4.ed). São Paulo: Autor.

Attux, C., Martini, L. C., Reis, A. F., \& Bressan, R. A. (2009). Intervenções não farmacológicas para manejo do ganho de peso em pacientes com esquizofrenia em uso de antipsicóticos. Arquivos Brasileiros de Endocrinologia \& Metabologia, 53(4), 391-398. Recuperado de http://www.scielo.br/pdf/abem/v53n4/v53n4a02. pdf. doi: $10.1590 /$ S0004-27302009000400002

Portaria $n^{\circ} 3.088$, de 23 de dezembro de 2011 . Institui a Rede de Atenção Psicossocial para pessoas com sofrimento ou transtorno mental e com necessidades decorrentes do uso de crack, álcool e outras drogas, no âmbito do Sistema Único de Saúde. Recuperado de http://bvsms.saude.gov.br/bvs/saudelegis/gm/2011/ prt3088_23_12_2011_rep.html

Portaria $n^{\circ} 336$, de 19 de fevereiro de 2002. Estabelecer que os Centros de Atenção Psicossocial poderão constituirse nas seguintes modalidades de serviços: CAPS I, CAPS II e CAPS III, definidos por ordem crescente de porte/complexidade e abrangência populacional, conforme disposto nesta Portaria. Recuperado de: http://bvsms.saude.gov.br/bvs/saudelegis/gm/2002/ prt0336_19_02_2002.html

Cardoso, C. S., Caiaffa, W. T., Bandeira, M., Siqueira, A. L., Abreu, M. N. S., \& Fonseca, J. O. P. (2006). Qualidade de vida e dimensão ocupacional na esquizofrenia: uma comparação por sexo. Cadernos de Saúde Pública, 22(6), 1303-1314. Recuperado de http:// www.scielo.br/pdf/csp/v22n6/19.pdf. doi: 10.1590/ $\underline{\text { so102-311X2006000600019 }}$

Carvalho, M. R. S., Silva, J. R. S., Gomes, N. P., Andrade M. S., Oliveira J. F., \& Souza M. R. R. (2017). Motivações e repercussões do consumo de crack: $\circ$ discurso coletivo de usuários de um Centro de Atenção Psicossocial. Escola Anna Nery, 21 (3), e20160178. Recuperado de http://www.scielo.br/pdf/ean/v2 ln3/pt_14148145-ean-2177-9465-EAN-2016-0178.pdf. doi: $\underline{10.1590 / 2177-9465 \text {-ean-2016-0178 }}$
Cavalcanti, M. T., Dahl, C. M., Carvalho, M. C. A., \& Valencia, E. (2009) Critérios de admissão e continuidade de cuidados em centros de atenção psicossocial, Rio de Janeiro, RJ. Revista de Saúde Pública, 43(Suppl 1), 23-28. Recuperado de http://www.scielo.br/ $\mathrm{pdf} / \mathrm{rsp} / \mathrm{v} 43 \mathrm{~s} 1 / 757 . p d f$. doi: 10.1590/s0034$\underline{89102009000800005}$

Cesari, L., \& Bandeira, M. (2010) Avaliação da qualidade de vida e percepção de mudança em pacientes com esquizofrenia. Jornal Brasileiro de Psiquiatria, 59(4), 293-301. Recuperado de http://www.scielo.br/ pdf/jbpsiq/v59n4/05.pdf. doi: 10.1590/s0047. $\underline{20852010000400005}$

Corrêa, M. M., Tomasi, E., Thumé, E., Oliveira, E. R. A., \& Facchini, L. A. (2017). Razão cintura-estatura como marcador antropométrico de excesso de peso em idosos brasileiros. Cadernos de Saúde Pública, 33(5), e00195315. Recuperado de http://www.scielo.br/

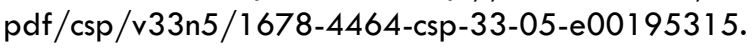
pdf. doi: $10.1590 / 0102-311 \times 00195315$

Coutinho, L. M. S., Matijasevich, A., Scazufca, M., \& Menezes, P. R. (2014) Prevalência de transtornos mentais comuns e contexto social: análise multinível do São Paulo Ageing \& Health Study (SPAH). Cadernos de Saúde Pública, 30(9), 1875-1883. Recuperado de http://www.scielo.br/pdf/csp/v30n9/0102$311 \mathrm{X}-\mathrm{csp}-30-9-1875$.pdf. doi: 10.1590/0102$311 \times 00175313$

Cristóvão, M. F., Sato, A. P. S., \& Fujimori, E. (2011). Excesso de peso e obesidade abdominal em mulheres atendidas em Unidade da Estratégia Saúde da Família. Revista da Escola de Enfermagem da USP, 45(spe2), 1667-1672. Recuperado de http:// www.scielo.br/pdf/reeusp/v45nspe2/05.pdf. doi: 10.1590/S0080-62342011000800005

Darviri, C., Artemiadis, A. K., Tigani, X., \& Alexopoulos, E. C. (2011) Lifestyle and self-rated health: a cross-sectional study of 3,601 citizens of Athens, Greece. BMC Public Health, 11:619. Recuperado de https://bmcpublichealth.biomedcentral.com/ articles/10.1186/1471-2458-11-619. doi: 10.1186/1471-2458-11-619

Franzmann, U. T., Kantorski, L. P., Jardim, V. M. R., Treichel, C. A. S., Oliveira, M. M., \& Pavani F. M. (2017). Fatores associados à percepção de melhora por usuários de Centros de Atenção Psicossocial do Sul do Brasil. Cadernos de Saúde Pública, 33(7), e00085216. Recuperado de http://www.scielo.br/pdf/csp/ v33n7/1678-4464-csp-33-07-e000852 16.pdf. doi: $10.1590 / 0102-311 \times 00085216$ 
Freitas, P. H. B., Pinto, J. A. F., Nunes, F. D. D., Souza, A. R. S., Machado, R. M. (2016). Esquizofrenia refratária: qualidade de vida e fatores associados. Acta Paulista de Enfermagem, 29(1), 60-68. Recuperado de http://www.scielo.br/pdf/ape/ v29n1/1982-0194-ape-29-01-0060.pdf. doi: $\underline{10.1590 / 19820194201600009}$

Kengeriski, M. F., Oliveira, L. D., Delgado V., \& Escobar, M. (2014). Estado nutricional e hábitos alimentares de usuários em Centro de Atenção Psicossocial de Porto Alegre, Brasil. Clinical \& Biomedical Research, 34(3), 253-259. Recuperado de https://seer.ufrgs.br/hcpa/ article/view/46806/31331

Leitão-Azevedo, C. L., Abreu, M. G. B., Guimarães, L. R., Moreno, D., Lobato, M. I., Gama, C. S., \& Belmontede-Abreu, P. S. (2006). Sobrepeso e obesidade em pacientes esquizofrênicos em uso de clozapina comparado com o uso de outros antipsicóticos. Revista de Psiquiatria do Rio Grande do Sul, 28(2), 120 128. Recuperado de http://www.scielo.br/pdf/ rprs/v28n2/v28n2a04.pdf. doi: 10.1590/S0101$\underline{81082006000200004}$

Leite, I. C., Valente, J. G., Schramm, J. M. A., Daumas, R. P., Rodrigues, R. N., Santos, M. F., ... Mota, J. C. (2015). Carga de doença no Brasil e suas regiões, 2008. Cad. Saúde Pública, 31 (7), 1551-1564. Recuperado de http://www.scielo.br/pdf/csp/v31n7/0102$311 \mathrm{X}$-csp-31-7-1551.pdf. doi: 10.1590/0102$311 \times 00111614$

López-Sobaler, A. M., Aparicio, A., Aranceta-Bartrina, J., Gil, Á., González-Gross, M., Serra-Majem, L., ... Ortega, R. M. (2016). Overweight and General and Abdominal Obesity in a Representative Sample of Spanish Adults: Findings from the ANIBES Study. BioMed Research International, 2016:8341487. Recuperado de https:// www.ncbi.nlm.nih.gov/pubmed/27382572. doi: $10.1155 / 2016 / 8341487$

Medeiros, C. R. O., Possas, M. C., \& Valadão Júnior, V. M. (2018). Obesidade e organizações: uma agenda de pesquisa. REAd - Revista Eletrônica de Administração, 24(1), 61-84. Recuperado de http://www.scielo.br/ $\mathrm{pdf} / \mathrm{read} / \mathrm{v} 24 \mathrm{n} 1 / 1413-2311$-read-24-1-61.pdf. doi: 10.1590/1413-2311.173.63838

Organização Mundial da Saúde. (1996). Classificação Estatística Internacional de Doenças e Problemas Relacionados à Saúde: CID-10 Décima revisão (Vol 2, $3 a$ ed). São Paulo: EDUSP.

Organização Mundial da Saúde/The World Bank (2012). Relatório Mundial sobre a Deficiência. São Paulo: SEDPcD. Recuperado de http://www. pessoacomdeficiencia.sp.gov.br/usr/share/documents/ RELATORIO_MUNDIAL_COMPLETO.pdf
Ramos, R., Karollyne, D., Guimarães, J., \& Yasui, Silvio (2011). Rupturas e encontros: desafios da Reforma Psiquiátrica brasileira. Saúde em Debate, 35(89), 341 -342. Recuperado de http://www.redalyc.org/ pdf/4063/406344813019.pdf

Rocha, S. V., Almeida, M. M. G., Araújo, T. M., \& Virtuoso Júnior, J. S. (2010). Prevalência de transtornos mentais comuns entre residentes em áreas urbanas de Feira de Santana, Bahia. Revista Brasileira de Epidemiologia, 13(4), 630-640. Recuperado de http://www.scielo. $\mathrm{br} / \mathrm{pdf} / \mathrm{rbepid} / \mathrm{v} 13 \mathrm{n} 4 / 08 . p d f$. doi: 10.1590/S1415790X2010000400008

Santos, E.G., \& Siqueira, M. M. (2010). Prevalência dos transtornos mentais na população adulta brasileira: uma revisão sistemática de 1997 a 2009. Jornal Brasileiro de Psiquiatria, 59(3), 238-246. doi: 10.1590/S0047-20852010000300011

Scholl, C. C., Tabeleão, V. P., Stigger, R. S., Trettim, J. P., Mattos, M. B., Pires, A. J., ... Quevedo, L. A. (2017). Qualidade de vida no Transtorno ObsessivoCompulsivo: um estudo com usuários da Atenção Básica. Ciência \& Saúde Coletiva, 22(4), 13531360. Recuperado de http://www.scielo.br/pdf/ csc/v22n4/1413-81 23-csc-22-04-1353.pdf. doi: 10.1590/1413-81232017224.02062015

Silveira, M. F., Almeida, J. C., Freire, R. S., Haikal, D. S., \& Martins, A. E. B. L. (2013). Propriedades psicométricas do instrumento de avaliação da qualidade de vida: 12 -item health survey (SF-12). Ciência \& Saúde Coletiva, 18(7), 1923-1931. Recuperado de http:// www.scielo.br/pdf/csc/v18n7/07.pdf. doi: 10.1590/ $\underline{\text { S1413-81232013000700007 }}$

Susce MT, Villanueva N, Diaz FJ, \& Leon J. (2005). Obesity and associated complications in patients with severe mental illness: a cross-sectional survey. J Clin Psychiatry, 66(2), 167-73. Recuperado de https://www.ncbi.nlm. nih.gov/pubmed/15705001

Verdolin, L. D., Borner A. R. S., Guedes Junior, R. O., Silva, T. F. C., \& Belmonte T. S. A. (2012). Comparação entre a prevalência de transtornos mentais em pacientes obesos e com sobrepeso. Revista Scientia Medica, 22(1), 25-31. Recuperado de http:// revistaseletronicas.pucrs.br/ojs/index.php/ scientiamedica/article/viewFile/9918/7531

Viana, M. C., \& Andrade, L. H. (2012). Lifetime Prevalence, age and gender distribution and age-of-onset of psychiatric disorders in the São Paulo Metropolitan Area, Brazil: results from the São Paulo Megacity Mental Health Survey. Rev Bras Psiquiatr, 34(3), 249-260. Recuperado de https://www.ncbi.nlm. nih.gov/pubmed/23429770. doi: 10.1016/i. rbp.2012.03.001 
World Health Organization. (2011). Waist circumference and waist-hip ratio: report of a WHO expert consultation, Geneva, 8-1 1 December 2008. Geneva, Switzerland: Autor. Recuperado de http://apps.who.int/iris/ bitstream/handle/10665/44583/9789241501491_ eng.pdf? sequence $=1$

World Health Organization. (2000). Cross-national comparisons of the prevalences and correlates of mental disorders. Bulletin World Health Organization, 78(4), 413-26. Recuperado de https://www.ncbi.nlm. nih.gov/pubmed/10885160

World Health Organization. (2002). Prevention and promotion in mental health. Geneva: World Health Organization. Recuperado de http://www.who.int/mental_health/ media/en/545.pdf

Zhang Z-q, Deng J, He L-p, Ling W-h, Su Y-x, Chen Y-m (2013). Comparison of Various Anthropometric and Body Fat Indices in Identifying Cardiometabolic Disturbances in Chinese Men and Women. PLOS ONE, 8(8), e70893. Recuperado de https://www.ncbi.nlm. nih.gov/pmc/articles/PMC3741370/. doi: 10.1371/ journal.pone.0070893

Zortéa, K., Bock P. M., Moreno, D. B., \& Abreu, P. S. B. (2009). Avaliação antropométrica e bioquímica em pacientes com esquizofrenia usuários de clozapina. Revista de Nutrição, 22(5), 697-705. Recuperado de http:// www.scielo.br/pdf/rn/v22n5/v22n5a10.pdf. doi: $10.1590 / \mathrm{S} 1415-52732009000500010$

Zortéa, K., Guimarães, L. R., Gama, C. S., \& Belmonte-deAbreu, P. S. (2010) Estado nutricional de pacientes com esquizofrenia frequentadores do Centro de Atenção Psicossocial (CAPS) do Hospital de Clínicas de Porto Alegre. J Bras Psiquiatr, 59(2), 126-130. Recuperado de http://www.scielo.br/pdf/ibpsiq/ v59n2/a08v59n2.pdf. doi: 10.1590/s0047$\underline{20852010000200008}$ 